\title{
An improved approach for robust road marking detection and tracking applied to multi-lane estimation.
}

\author{
Marc Revilloud, Dominique Gruyer IEEE Member, Evangeline Pollard
}

\begin{abstract}
In this paper, an original and innovative algorithm for multi-lane detection and estimation is proposed. Based on a three-step process, (1) road primitives extraction, (2) road markings detection and tracking, (3) lanes shape estimation. This algorithm combines several advantages at each processing level and is quite robust to the extraction method and more specifically to the choice of the extraction threshold. The detection step is so efficient, by using robust poly-fitting based on the point intensity of extracted points, that correction step is almost not necessary anymore. This approach has been used in several project in real condition and its performances have been evaluated with the sensor data generated from SiVIC platform. This validation stage has been done with a sequence of 2500 simulated images. Results are very encouraging : more than $95 \%$ of marking lines are detected for less than $2 \%$ of false alarm, with $3 \mathrm{~cm}$ accuracy at a range of $60 \mathrm{~m}$.
\end{abstract}

\section{INTRODUCTION}

For at least two decades, the development of transportation systems have led to the developement of embedded applications allowing to improve the driving comfort and to minimize the risk level of hazardous areas. More specificaly, the researches in intelligent and Advance Driving Assistance Systems have provided a great number of devices on many types of automatic vehicle guidance and security systems such as obstacle detection and tracking [1], road visibility measurement [2], pedestrian detection, road departure warning systems.... However, one of the first embedded system that was studied is probably the lane detection system. This application is usually based on road marking detection algorithms. [3]. This system is also one of the most important source of information in order to build a local perception map of an environment surrounding an ego-vehicle. Indeed, this information provides relative vehicle location information to all other perception systems (obstacles, road signs, ...) that need to know the road and lanes attributes. For this reason the system must be as robust as possible. Moreover, for several year, it appears evident the automation of the driving task is probably a solution in the reduction of the road injuries. But for automated or partially automated driving task, the road marking and lane localization are very important and provide a critical information. This information needs to be really accurate, certain, reliable in order to achieve some manoeuvers like lane changes or generate safe path planing (co-pilot) [4].

M. Revilloud and D. Gruyer are with the LIVIC research laboratory, IFSTTAR, 14 route de la Miniére, bat 824, 78000 Versailles, France

E. Pollard is with the INRIA Paris-Rocquencourt research laboratory, in the project-team IMARA evangelinepollardegmail.com
The research and the study proposed in this paper are directly dedicated to this important topic of road markings detection and tracking, and lanes estimation for automated and/or partialy automated driving applications. Our objective is to provide an assessment of the road surface attributes (road markings attributes, type of road marking, number of lane and characteristic of lanes). This method is based on use of one or several embedded cameras.

Most of the algorithms are basically based on a three-step scheme summarized as follows. First, images are processed in order to extract road marking features. Second, extracted primitives are analyzed in order to extract point distributions corresponding to a road marking. And finally in a third step, extracted and validated points are used to extract lane shape. In some previous work [5], the first extraction part has been studied, tested, and evaluated in order to determine the best way to extract road marking primitives. In this paper, a double extraction strategy is proposed to achieve the discimination of the points for marking points and nonmarking points. To guaranty the robustness of our approach, we proposed in addition a performance evaluation protocol for the first road primitives extraction stage based on the use of the SiVIC platform, which is presented in [6]. This protocol provides an accuracy measurement of the clustering and robustness relatively to a clustering threshold $T_{g}$.

In this paper, we present several significant improvements of the original method proposed by S. S. Ieng and D. Gruyer in [7]. The global scheme is the same one but some enhancements have been done in each part. For instance, the combination strategy of several extractors, the management of the primitives in the detection stage, and the lane and markings estimation in the lane estimation part has been modified. In addition, instead of imposing a very discriminative threshold into the extraction part, we propose the use of the intensity of the extracted point into both the detection and the estimation parts. Lane marking detection, originally based on the study of an histogram containing projected points, is now made by using the same type of histogram but where the projected point are weighted in function of their uncertainty. Moreover, the poly-fitting mechanism has been replaced by a a weighted poly-fitting, for the same reason. Higher is the extracted intensity points, more strongly weighted are these points in the estimation process. To robustify our approach and avoid false alarms, distribution points which are not satisfying very discriminative criteria for peak clustering are submitted to a robust weighted polyfitting. In this way, point distributions containing outliers (as it could happen in the case of sidewalks or guardrail which 
produce false alarms), are still validated as road markings. Finally, comparatively to the initial version of this algorithm, the proposed improvements lead to better results. These changes in the different part of this method allow to affirm that the use of the filtering step is not important anymore.

The Fig. 1 gives an global overview of the proposed road marking detection and tracking and lane estimation architecture. The paper is organized as follows. In Sec. II, the

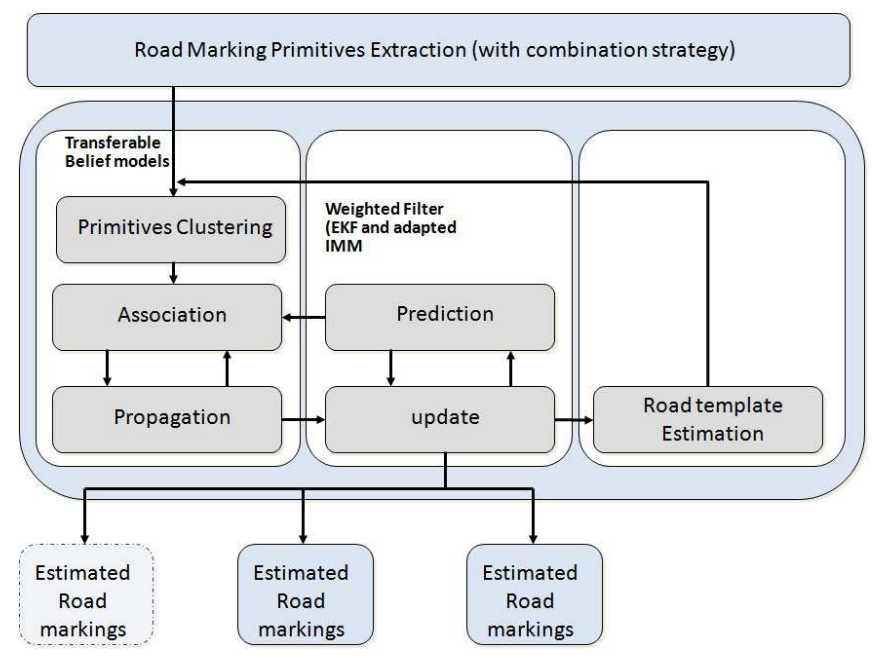

Fig. 1. The entire process for road marking detection

SiVIC platform used for the evaluation of marking detection is presented. In Sec. III, the three main parts of this approach are detailed. Sec. IV described the evaluation protocol used for road marking detection algorithm. Sec. V presents a set of results with differents road conditions and levels of quality for the road markings.

\section{THE SIVIC PLATFORM}

SiVIC is a virtual sensor simulation platform for ADAS prototyping. It simulates different embedded sensors (camera, telemeters, GPS, radar, communication devices, INS, odometer ...) and the dynamic vehicle behaviour of vehicle as described in [6]. In order to prototype and to test ADAS, a realistic 3D reproduction of the real Satory's test track was produced by LIVIC. Fig. 2 shows the similarity of this simulation in comparison with the same perspective on the real track. In this figure, the first line provides virtual renderings and the second line gives the real pictures from the same point of view. This simulation takes into account a great number of detail such as as the road shape, guard rails, buildings, road sign elements, trees, etc. The images generated by the SiVIC platform can be considered relatively close to the images which could be provided by a real camera Fig. 2. The quality of this virtual platform is mainly due to:

- The high accuracy of both the shapes of the Satory's track (build from a centimetric GPS-RTK) and the additional element of the environment (buiding, guardrail, sidewalk, trees, road signes, differents fences, ...)

- Natural images of bitumen used as road textures

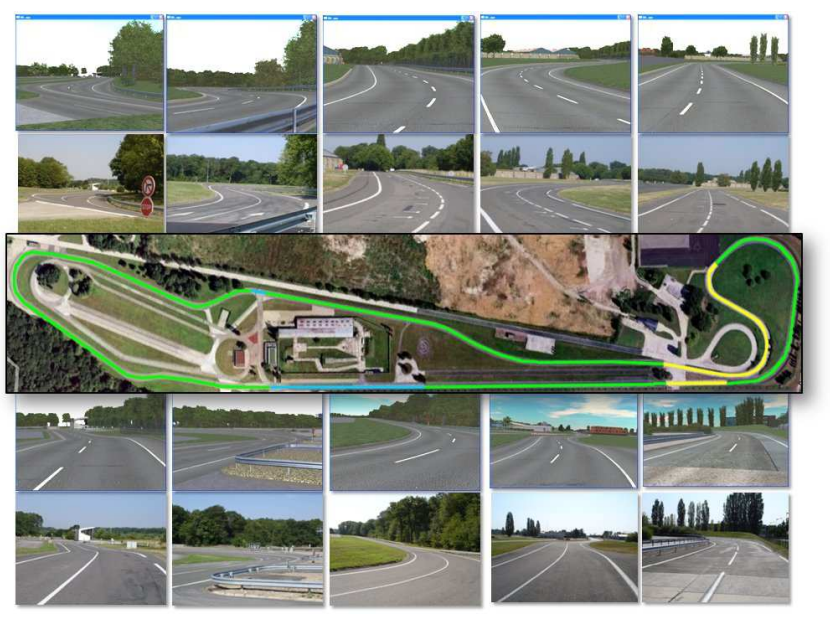

Fig. 2. Comparison of SIVIC and natural images

- The realistic model of the vehicle dynamics

- An accurate modeling of the optical sensor as describe in [8]

- The capabilities of SiVIC to provide both camera images and the associated ground truth.

\section{ROAD MARKING DETECTION AND ESTIMATION}

On the same principle as [7], a three step process for lane marking detection and estimation is proposed. The main purpose is to obtain the number of lane markers $N_{k}$, their position and their shape. For each validated lane marker $m$, the goal is also to provide an estimation of the road shape as a second degree polynomial in the vehicle coordinate system

$$
x=a_{0}^{m}+a_{1}^{m} \cdot y+a_{2}^{m} \cdot y^{2}
$$

As illustrated in Fig. 1, the first step consists in extracting road marking primitives. The second step is dedicated to the association stage. In fact from a set of extracted primitives, the objective is to detect the number of road markings and to associate them with the last ones (the road marking tracks). In a third step, given a set of labeled primitives, the goal is to provide, for each frame $k$, an estimation of the real state $A_{m, k}$ of any road marking $m$ :

$$
A_{m, k}=\left[a_{k, 0}^{m}, a_{k, 1}^{m}, a_{k, 2}^{m}\right]^{T}
$$

corresponding to eq. (1), where $(\cdot)^{T}$ denotes the transpose transformation. With this information, an associated uncertainty matrix $P_{m, k}$ is also estimated.

\section{A. Lane marker feature extraction}

The first step consists also in establishing which input image points belong to a lane marker. Most of the time, lane marker forms bright region (white or yellow paint) on a dark background (asphalt), having a limited width. Extraction purpose is also to detect regions with a gradient intensity higher than a certain threshold $T_{g}$ and bounded by the interval $\left[S_{m}, S_{M}\right]$. It has been shown in [9], that local thresholding methods provide the best results. In some previous work [10], a protocol was proposed for the evaluation of road marking 
extraction algorithms, based on synthesized images coming from simulator Sivic. After a study about performances of four extraction algorithms based on local thresholding, we proposed a new lane feature extraction algorithm based on a double extraction scheme. However, experimental results show that the assumption that better are the extraction step (using the procole used in [5]), more accurate is the road state estimation is uncorrect. Results presented in Sec. V prove that with the improvement of detection part, if double extraction scheme does not degrade performances, it does not improve it and necessarily increases time processing. Extraction step is also limited to SLT (Symmetric Local Threshold) in order to provide a set of extracted points $E$ and their corresponding intensity.

Finally, to enable the processing of extracted point, each extracted point of each cameras are projected in the vehicle coordinate system. This projection stage uses intrinsic and extrinsic parameters of the camera which are known. The position is fixed, perpendicularly to the car frame. The origin of the camera coordinate system $\mathcal{R}_{\text {cam }}=$ $\left(O_{\text {cam }}, \vec{X}_{c a m}, \vec{Y}_{\text {cam }}, \vec{Z}_{\text {cam }}\right)$ is also established at coordinates $(0,0, h)$ in the vehicle coordinate system, denoted $\mathcal{R}_{v}=$ $\left(O_{v}, \vec{X}_{v}, \vec{Y}_{v}, \vec{Z}_{v}\right)$, as illustrated in Fig. 3, with $h$ the height of the camera knowing the car reference. This coordinate system is centered on the car referential, perpendicularly to the road surface, assumed to be plane.

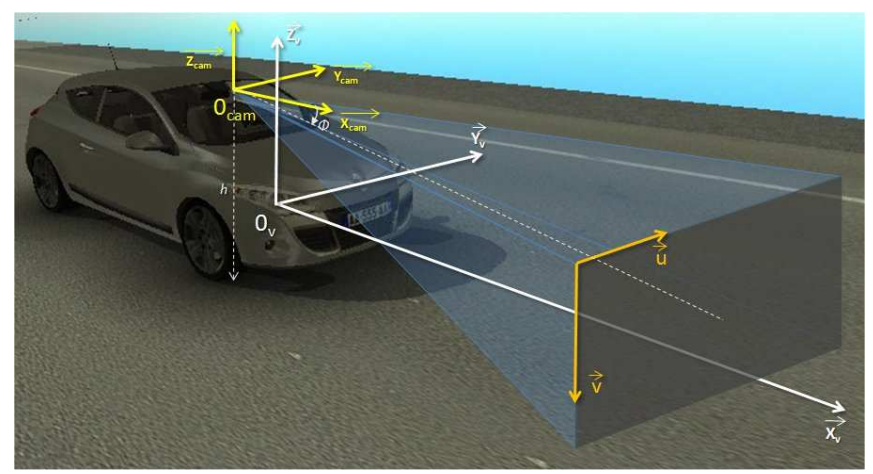

Fig. 3. Observation and coordinate system

\section{B. Lane marker detection}

1) Point projection: Knowing the set of extracted points $E$ ( $c f$. Fig. 4-(a)), the goal is now to detect marking lines and label extracted points according to detected lines. Their associated intensity is used in addition in order to take into account their uncertainty. Even if Hough transformation, traditionally used to detect straight lines can be extended to curves [11], the proposed approach is based on an analysis of the spatial distribution of extracted points on the $X_{v}$ axis. $X_{v}$ space is first cut into constant space intervals between $X_{v}^{\min }$ and $X_{v}^{\max }$. Contrary to [3], where points are projected along the $Y_{v}$ axis, our 2D-detected points are projected along the road shape on the $X_{v}$ axis. They are projected between 1 and 255 times depending on the value of their intensity. Shape of the road is established by using best available estimation states. More details are given to define what is a good estimation in Sec. III-F. According to its projection state $x_{i}^{p}$, each point $i(\forall i \in\{1, \ldots, n\})$ given by primitive extraction step illustrated in Fig. 4-(a) and projected to vehicle coordinate system, can be associated to a $x$-interval corresponding to a dynamic projection template, illustrated in Fig. 4-(b). A point density histogram is then constructed. To distinguish marking lines to false alarms and to precisely establish peak coordinates, the histogram is convoluted with a Normal distribution $\mathcal{N}\left(\cdot,\left(\sigma_{i}^{c}\right)^{2}\right)$ with a variance $\left(\sigma_{i}^{c}\right)^{2}$ corresponding to the width of two histogram intervals, as shown in Fig. 4-(c). Histogram peaks correspond to marking lines. The next step consists in precisely and reliably detect histogram peaks.

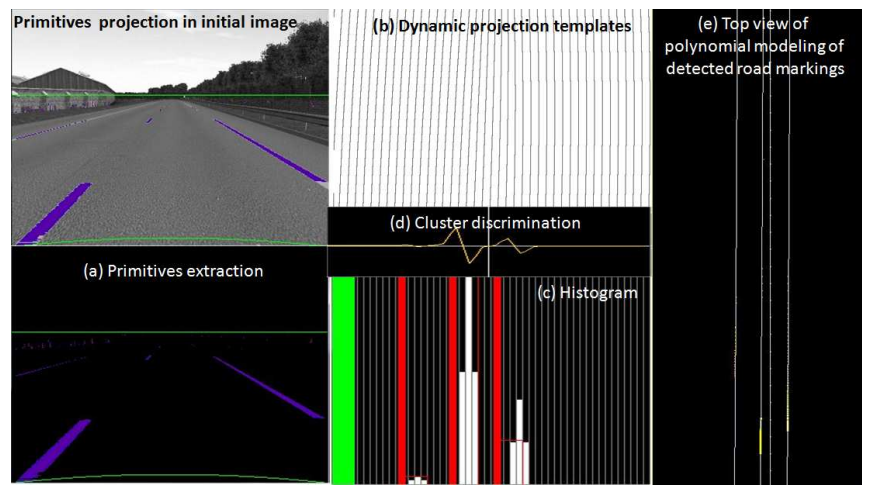

Fig. 4. Marking line detection

It has been shown in [12], that a non-Gaussian model is more adapted to describe perturbations on the observation of lane markers. Ieng et al. also propose to model noise measurements as a Smooth Exponential Family (SEF) distribution defined according parameters $\alpha$ and $\zeta$. The approach consists in detecting error minima by calculating zero crossing of the following third derivative (illustrated in Fig. 4-(d)) of the error function $\phi_{\alpha}$ :

$$
\frac{\partial^{3} \phi_{\alpha}}{\partial t^{3}}=\frac{4 t}{\zeta^{4}}\left(1+\frac{t^{2}}{\zeta^{2}}\right)^{(\alpha-3)}(\alpha-1)\left(3+\frac{t^{2}}{\zeta^{2}}(2 \alpha-1)\right)
$$

Zero crossing corresponds to the projected position $X_{m}^{p}$ of potential marking lines.

2) Peak validation: Peaks are first filtered according to the number of points: the number of points forming the peak must be higher than the minimum number of points $N_{\text {min }}$.

Sets of labeled points (labeled according to the peak label) are written under a matrix form as:

$$
X_{m, k}=\left[\begin{array}{ccc}
1 & x_{1} & x_{1}^{2} \\
\vdots & \vdots & \vdots \\
1 & x_{N_{m}} & x_{N_{m}}^{2}
\end{array}\right], Y_{m, k}=\left[\begin{array}{c}
y_{1} \\
\vdots \\
y_{N_{m}}
\end{array}\right]
$$

The polynomial fit of $A_{m, k}$, denoted $\tilde{A}_{m, k}$ is obtained by using a weighted least squares estimation as follows:

$$
\tilde{A}_{m, k}=\left(X_{m, k}^{T} W_{m, k} X_{m, k}\right)^{-1} X_{m, k}^{T} W_{m, k} Y_{m, k}
$$




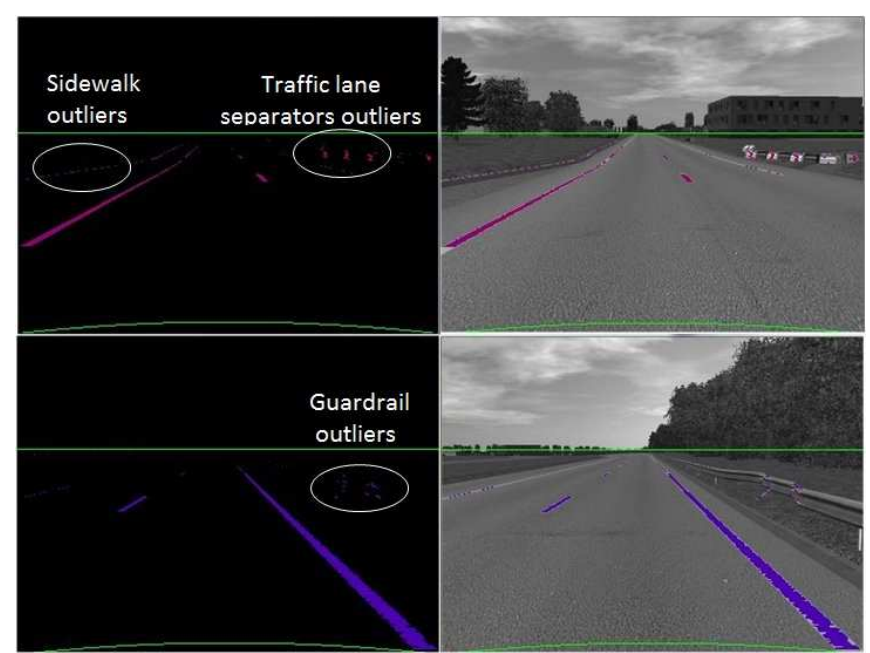

Fig. 5. Outliers due to a sidewalk

where $W_{m, k}$ is a diagonal matrix containing weight dedicated to each point. Here, the weight is calculated as the intensity point. Several criteria are here tested. The associated variance must be smaller than the maximum variance $\sigma_{\max }$. The average intensity point must be higher than a threshold $\gamma_{\min }$. The curvature $\left(a_{k, 2}^{m}\right)$ must be smaller than the maximum road curvature coming from the route code $\theta_{\max }$. The $y$-intercept $\left(a_{k, 0}^{m}\right)$ must be smaller than a given distance $d^{0}$ (lane markings which are too far away from the vehicle cannot be detected).

Due to the inversion of a $N_{m} \times N_{m}$ matrix, poly-fitting step can be time consuming. However, following [13], this computation is largely optimized to respect real-time constraints.

If the associated variance is higher than the maximum variance $\sigma_{\max }$, then a robust poly-fitting estimation is tested in order to discriminate cases where a lot of outliers occur. It can happen for example if there is a sidewalk along the road or a traffic barrier on the highway as illustrated on Fig. 5 .

Due to its robustness to a high number of outliers, Ransac method is currently used for robust poly-fitting [14]. However, there is no upper bound in processing time and in the case presented in Fig. 5, it could converge toward the bad solution. On the observation that, the right point set is closer to the camera than the one generated by the sidewalk or the traffic barrier, we suppose that the number of points in the right set is higher. Assuming that the lane marking distribution points are distributed according to a Gaussian distribution, we simply proposed, from the first estimate, to calculate the association probability for each point, according to the standard deviation $\sigma^{r}$. Points with a probability lower than a given threshold $\gamma$ (this threshold is obtained from the inverse $\chi^{2}$ ) are eliminated. In this way, unlikely points are mainly eliminated and a second more precise estimate is then calculated.

Finally, according to this robust poly-fitting, the corresponding variance must be smaller than the maximum variance $\sigma_{\max }^{r}$.
As an output, a set of detected marking lines $\tilde{A}_{m}(\forall m<$ $M_{k}$ ) is obtained, with $M_{k}$ the number of detected marking lines at the current iteration $k$ as illustrated in Fig. 4-(e)).

\section{Lane marker association}

Now, an association step is necessary in order to establish relationship between the set of detected marking lines $\tilde{A}_{m}$ and the set of estimated marking lines $\hat{A}_{m, k-1} \forall(m<$ $N_{k-1}$ ) known from the previous iteration $k-1$. The proposed approach uses the Transferable Belief Model (TBM) framework [15]. This formalism, instead of manipulating probability distribution, manipulates belief mass distribution, also called basic belief assignment (bba). The belief mass is defined as the mass of elementary probability on an assumption $A$ :

$$
\begin{aligned}
m^{\Omega}: & 2^{\Omega} \rightarrow[0,1] \\
A & \rightarrow m^{\Omega}(A)
\end{aligned}
$$

This mass is calculated for each proposition $A$ of the referential defined as $2^{\Omega}=\{A \mid A \subseteq \Omega\}$ called the powerset. The power-set contains all singleton hypothesis, but also the union of hypotheses. The union of all hypotheses constitutes the doubt or the absence of knowledge. The data association is made following the Gruyer's and Royere's approach initially proposed in [16] and extended in [17]. The idea is to first calculate a prior bba for each peak-toline association, based on a similarity measure. Then, prior bbas are combined to calculate each association hypothesis belief. This operation is carried out twice in an extended open world: a fist time for the peak-to-line association allowing to manage the road marking appearance, and a second time for the line-to-peak association in order to detect the line disappearance.

The distance function used to quantified the similarity between a new detected road marking (histogram peak) and a road marking track (previous road marking) has been initially proposed by Gruyer [18] in order to calculate a similarity distance between two clusters taking into account their uncertainties (both the variance and co-variance of each cluster). This distance function is the same one used in the original algorithm [7], it is based on the computation of the outer clusters distances normalized by the sum of the inner distances.

\section{Lane marker certainty propagation}

As for obstacle tracking, it is important to ensure a temporal continuity in the association. The management of this temporal continuity is essential to estimate the dynamics of an object and to predict its future state. Furthermore, in order to quantify its relevance and temporal coherence, a certainty value called $c$ is assigned to each object. The way to update and propagate this certainty is illustrated in Fig. 6. At the previous iteration $k-1$, an estimated marking line $\hat{A}_{m, k-1}$ has a current certainty denoted $c\left(\hat{A}_{m, k-1}\right)$ (case 1$)$. The certainty is then projected on the propagation function (case 2 on the blue line), in order to obtain a normalized corresponding number of iterations (case 3 ). In case 4.1, 


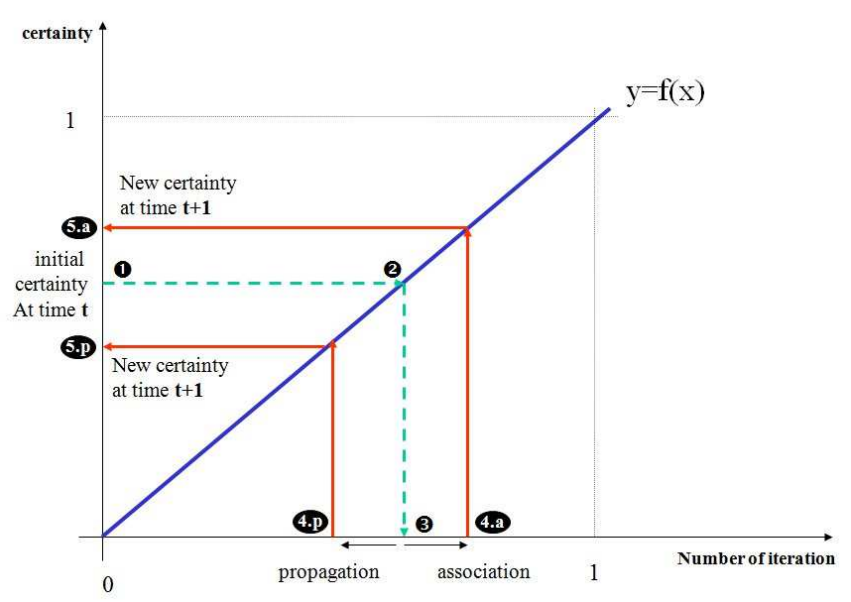

Fig. 6. Certainty with $n^{\prime}$ the normalized number of iterations

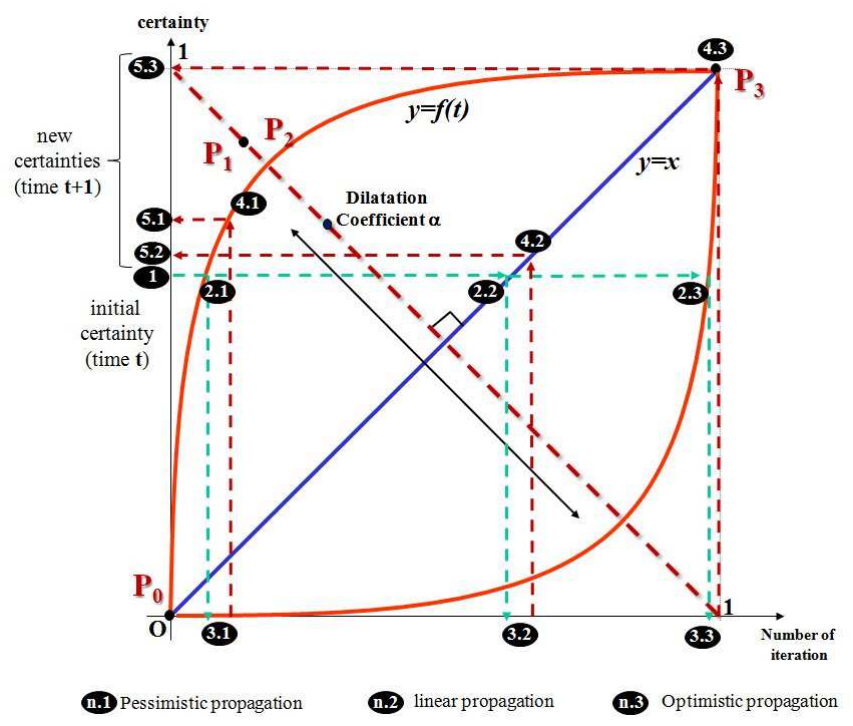

Fig. 7. Certainty propagation

the estimated object $\hat{A}_{m, k-1}$ is associated to a detected object $\tilde{A}_{m^{\prime}}$. In this case, the corresponding relative number of iterations increases relatively to both the belief into this association $m_{1 \ldots M_{k}}^{\Omega_{M_{k}}^{\star}}\left\{\hat{A}_{m, k-1}\right\}\left(\tilde{A}_{m^{\prime}}\right)$ and the quality of the road marking detection. This corresponding normalized number is yet reprojected following the same propagation function to obtain the new increased certainty $c\left(\hat{A}_{m, k}\right)$ at iteration $k$ (case 5.1). In the case where the estimated line marking is not associated, then the corresponding normalized number of iterations decreases by 1 (case 4.1) and the corresponding certainty $c\left(\hat{A}_{m, k}\right)$ also decreases. Each detected object is initialized with a certainty $c_{0}$. In the current method, this value is fixed to 0.5 but it will be clever to initialize this value in function of the quality of the current road marking detection.

In the Fig. 6, the certainty propagation is made with a linear fonction (with a slope of 1). However, in a more generic approach,this function is chosen depending on a coefficient $\alpha$ which is fixed relatively to the data reliability and confidence. The ability to choose a specific function, in order to propagate a certainty, is done to model both the optimistic and pessimistic behaviors of the certainty propagation as shown in Fig. 7. In the optimist case, the certainty increases quicker than in the pessimist case. The propagation function must be chosen symmetrical, continuously increasing and derivable. In this application, the used propagation function is a Bezier curve as proposed in [16].

When the certainty $c\left(\hat{A}_{m, k}\right)$ decreases until 0 , the object is deleted.

\section{E. Lane marker estimation}

Assuming that each road marking (lane marker) $m$ is now detected with a certainty $c\left(\hat{A}_{m, k}\right)$ and a set of labeled points $\left\{x_{i}, y_{i}\right\}_{\forall i \in\left\{1, \ldots, N_{m}\right\}}$ converted into the vehicle coordinate system, the goal is to provide an estimation $\hat{A}_{m, k}$ of the road marking shape as a second degree polynomial function as described in eq. 2.

Lane markers are not strictly constant over time and across successive images. They can be simply estimated using a linear Kalman filter, whom assumptions are now described. However, they change relatively slowly assuming a constant vehicle speed and under flat road assumption (required assumption for the point conversion into the vehicle coordinate) following the state equation:

$$
A_{m, k+1}=F_{k} \cdot A_{m, k}+\nu_{m, k}
$$

where $F_{k}$ designates the evolution matrix, $A_{k, m}$ the real road marking state at iteration $k$ and $\nu_{m, k}$ the model noise representing the uncertainty in the road marking evolution over time. If some authors like [19] uses elaborated and complex evolution matrix, we assume that the change between two successive image is negligible (matrix $F_{k}$ is equal to the identity matrix) and can be modeled thanks to the model noise, which must be adequately chosen. The model noise $\nu_{m, k}$ can be estimated by studying the maxium evolution of parameters of a road marking between iteration $k$ and $k+1$.

Measurement-to-state equation can be simply written as:

$$
X_{k, m} \cdot A_{k, m}=Y_{k, m}+w_{k, m}
$$

where $w_{k, m}$ represents the measurement noise estimated at each iteration as the error of one pixel shift in the vehicle coordinate system.

Assuming that extracted features cannot be modeled as Gaussian distribution, Ieng et al. estimates lane marker states by using a Robust Kalman Filter [1]. We propose as an alternative to use a gating computation [2] and to reestimate road marking shape $\tilde{A}_{m, k}$. A gate is also designed around the predicted position at iteration $k$ of road marking $\hat{A}_{k \mid k-1, m}$, based on the maximum acceptable measurement error according to the prediction error magnitude. Only points that are within the track gate are considered for update the lane marker at iteration $k$. As shown in Fig. 8, false points are filtered and selected points can be modeled as a Gaussian distribution.

At this stage, $\tilde{A}_{m, k}$ are so close to the reality that a corrective step is unnecessary and $\hat{A}_{m, k}=\tilde{A}_{m, k}$. 


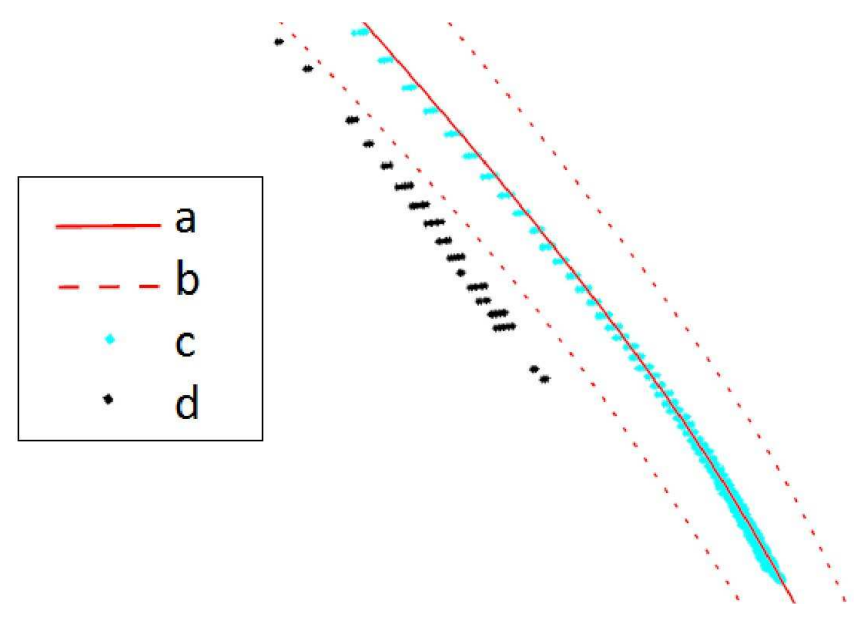

Fig. 8. Gating process illustration

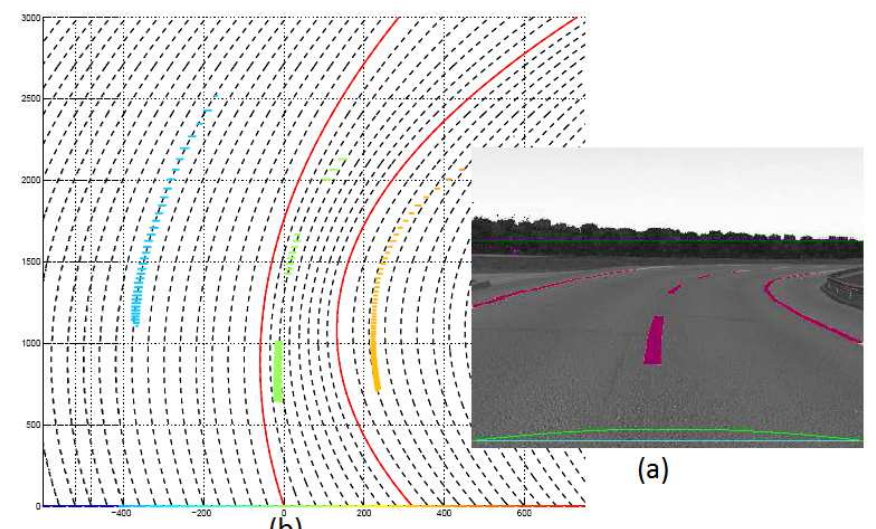

(b)

Fig. 9. Road shape interpolation in case of high curvature. (a) original images and its extracted points. (b) Interpolated road shape for each interval (red lines: previous road shape estimates, dotted lines: interpolated road shape). Points are colored accordingly to their interval.

\section{F. Road shape update}

As explained in Sec. III-B, extracted points are projected according to the global road shape. One solution consists in selecting the best road marking estimation (best to the point of view of varying criteria such as the number of extracted points, and the smallest error estimation) in order to project the points (road marking primitives) for the next iteration. However, in case of strong curvatures, we can note that the inside road markings have a stronger curvature than the outside ones as illustrated in Fig. 9-(a). The proposed solution consists in selecting several lane markers as road shape reference. Road markings are selected if they satisfy strict criteria (more strict than detection criteria) on:

- the number of points forming the peak must be higher than the minimum number of points $N_{\min }^{p}\left(N_{\min }^{p}>\right.$ $N_{\text {min }}$ ),

- the variance of the peak must be smaller than the maximum variance $\sigma_{\max }^{p}\left(\sigma_{\max }^{p}<\sigma_{\max }\right)$.

If several road marking estimates are selected as road shape reference, they are first tested to detect cross road

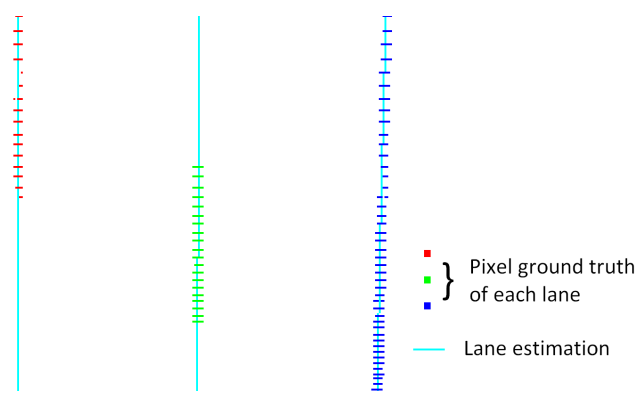

Fig. 10. Evaluation protocol

markings (cross lane markers). In fact, they are not supposed to cross each other. If road marking crossing is detected, only the lane marker with the minimum variance is selected. They are then used to interpolate a second degree polynomial function for each interval as illustrated in Fig.9-(b).

\section{Evaluation Protocol}

To evaluate the quality of this algorithm and the impact of each parameter, an evaluation tool and a protocol have been developed. The evaluation protocol uses the estimates from the lane detection algorithm and ground truth images provided by SiVIC as illustrated in Fig.10. This protocol is based on four criteria:

- Detection rate: Rate of good detection estimated according to the ground truth for one lane. This rate corresponds to the line ratio where distance between the estimate and the ground truth is below a given threshold $d_{\min }$. For the 1 line of our image, this distance $e_{m, k, l}$ between the estimate $\hat{A}_{m, k, l}$ and the the average points of the line $\overline{T_{m, k, l}}$ is calculated as an Euclidian distance:

$$
e_{m, k, l}=d\left(\hat{A}_{m, k, l}, \overline{T_{m, k, l}}\right)
$$

where $d(.,$.$) the euclidian distance. This threshold d_{\min }$ was chosen after empirical testing of different ADAS system.

- Precision: Accuracy in the case of correct detection for one lane. This accuracy is calculated as the mean of each $d(l)$ for each line of the ground truth image.

- False alarm rate: Ratio between the number of false detections and the real number of lanes.

- Global detection rate: Ratio between the number of correct detections and the real number of lanes.

\section{RESULts}

In this section, some results are presented according to the dedicated performance protocol developped with the SiVIC platform. This evaluation has been done on a Sivic sequence of images combining several situation: straight line, strong curvature, guardrails, sidewalks, fences, trees, different conditions of shadows... Detection rate (DR) and false alarm rate (FAR) quantify the quality of the detection. Detection rates per lane marking and their associated error measure the quality of the estimation.

Numerous parameters are used in this multi-lane detection method, which is determined before this evaluation step 

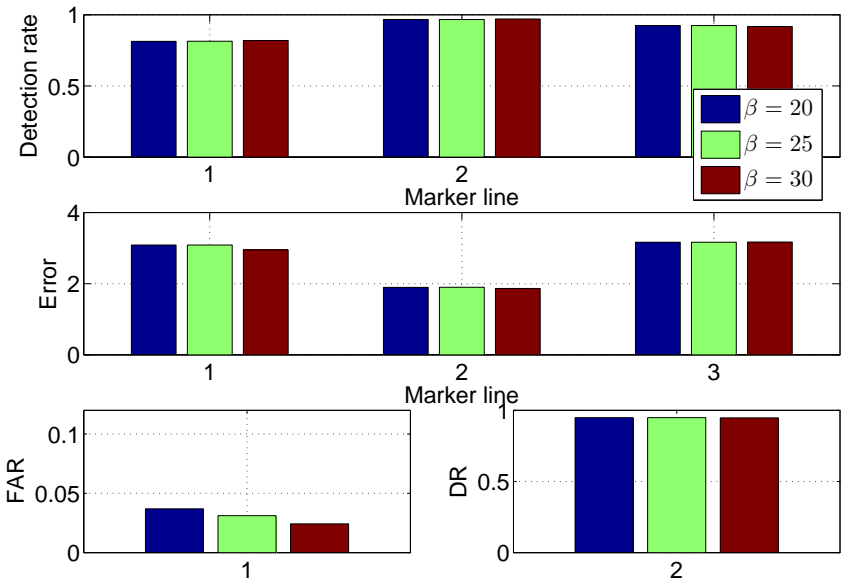

Fig. 11. Influence of the threshold on the average point intensity

using real and simulated image sequences. Except the minimum number of points for a peak to be validated (which depends on the image size), a great part of these parameters are independent of the road conditions. The minimum number of points $N_{\min }$ is fixed equal to 100 . Concerning the peak validation, the average point intensity threshold $\beta_{\text {min }}$ has been tested in Fig. 11. The best results are obtained with $\beta_{\min }=30$ and the threshold only influence FAR.

The maximum road curvature comes from the French regulations of road infrastructure as $\theta_{\max }=1.6 e^{-4}$ and we limit detection to a distance $d^{0}=8 \mathrm{~m}$.

Coefficients for the Smooth Exponential Family (SEF) distribution are defined as $\alpha=0.01$ and $\zeta=0.1$.

Poly-fitting parameters have been established as: $\sigma_{\max }=$ $50, \sigma_{\max }^{r}=100$ and $\sigma^{r}=400$.

Extraction parameters such as minimum and maximum width for lane marking are set to $\left[S_{m}, S_{M}\right]=[10,50]$ pixels. We use as an extraction threshold $T_{g}=5$ to limit time computation. Influence of this threshold is studied in Fig. 12. In this experiment, influence of the threshold is conjointly studied to the influence of the histogram with weighted points or not. We observe that with the weighted histogram, results are quite stable, whatever the value of the threshold, while with the unweighted histogram, threshold highly influences the quantity of false alarm.

As an estimation method, different model noises have been tested for a Kalman filter, but due to the high detection quality, best results are obtained without correction step and the impact of the Kalman filtering is not significant in the tested scenario. An extended evaluation will be necessary in order to confirm the uselessness of Kalman filter.

A robust weighted poly-fitting has been proposed in Sec. III-B.2. Different way to calculate the weight matrix $W_{k, m}$ have been tested: point intensity, distance to the vehicle and residual. 13 shows that best results are obtained with the intensity point.

In Fig. 14, several methods for the road update stage are tested: Several road marking estimates satisfying restrictive

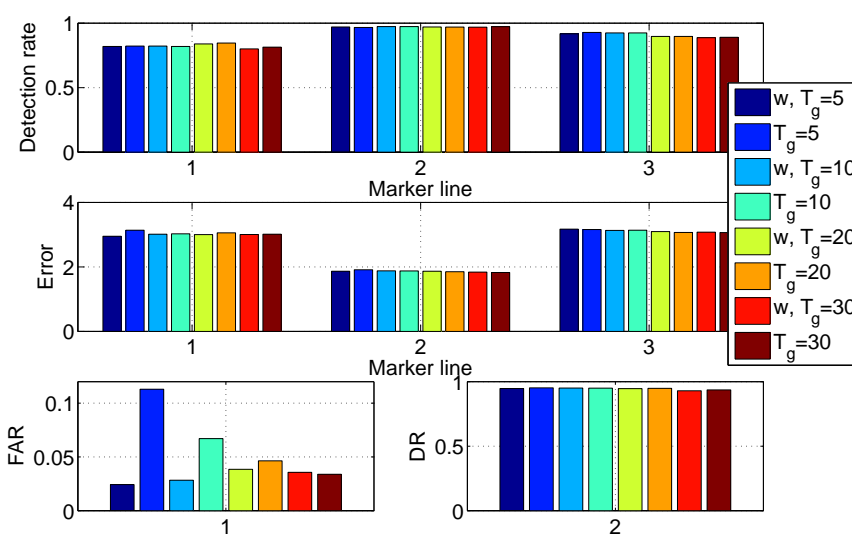

Fig. 12. Influence of extraction threshold $T_{g}$ by using intensity of extracted points or not (in legend, w means that weighted projection is used for histogram build)
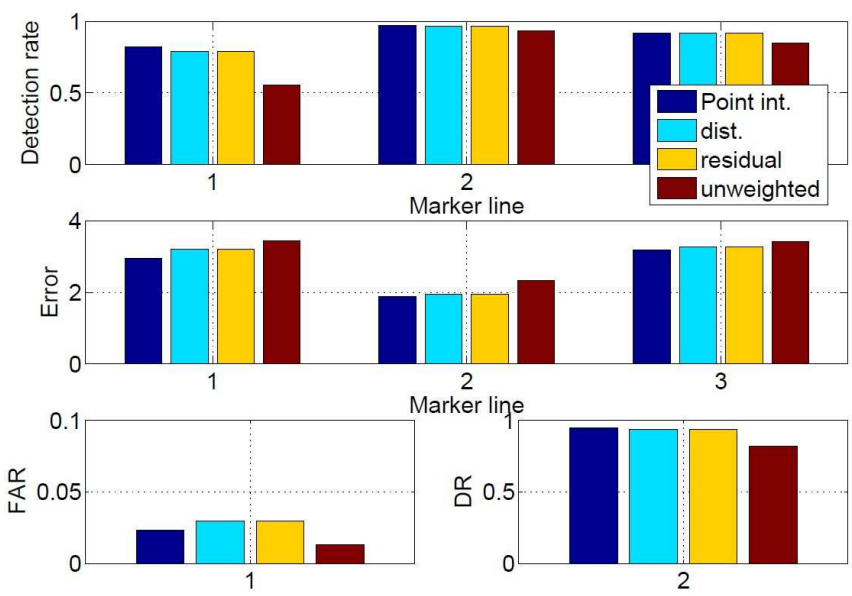

Fig. 13. Influence of robust weighted poly-fitting

constraint and best road marking estimate in terms of number of points or minimal variance. Best results are obtained when several road marking estimates are used, specially in case of strong curve.

\section{CONCLUSION}

In this paper, an original algorithm for multi-lane detection and estimation is proposed based on a three-step process, (1) extraction - (2) detection - (3) estimation. The algorithm is quite robust to the extraction method and more specifically to the choice of the extraction threshold. The detection step is so efficient, by using robust poly-fitting, that no estimation step is really necessary. Consequently, this algorithm respects the real-time constraint and produces very encouraging results: more than $95 \%$ of lane markers are detected for less than $2 \%$ of false alarm. Road shape estimate is close to the reality because the average error is less than $3 \mathrm{~m}$ for a range of $60 \mathrm{~m}$.

A new evaluation protocol also has been proposed. This protocol is based on synthesized images coming from the SIVIC simulator. It is the first time such a protocol is 

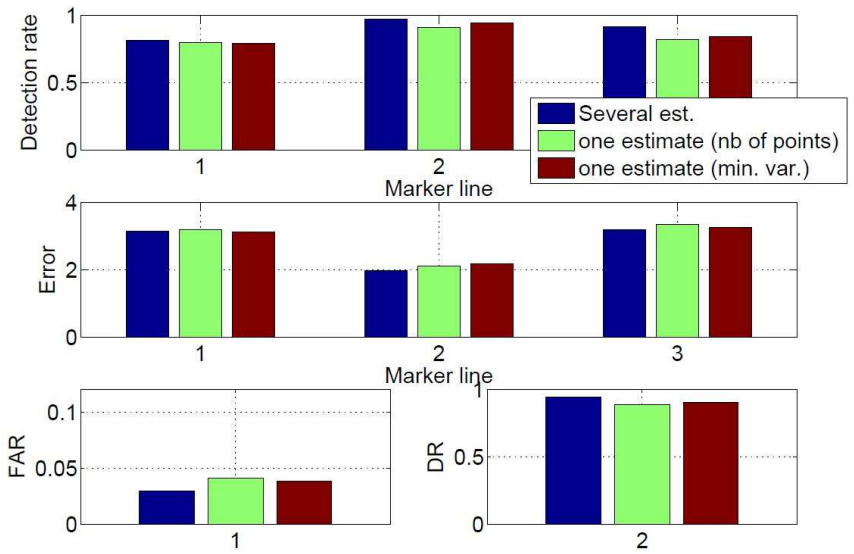

Fig. 14. Result with different road shape update

implemented with virtual data in order to validate a road marking detection method. The images provided by this platform are quite enough realistic to effectively validate an image processing application [8]. The evaluation protocol is based on three criteria: the detection rate of road markings (lane markers), the precision of their estimation and a false alarm rate. Numerous tests on a set of parameters prove the robustness of this approach. Finally, the proposed system has been tested with success on several hours of data collected on the real Satory's test tracks under varying lighting and curvature conditions. The real images recorded during these trials correspond to the same one generated with the SiVIC platform. The main goal was to obtain similar results and the same behavior in both the real and the simulated scenarios for autonomous driving applications. Unfortunatly, some difficulties can appear in the road marking detection when the vehicle is accelerating (due to the pitch) or when the flat road assumption is not respected, but this should be solved by integrating information on the pitch into the data conversion stage (image space toward 1D space).

To improve the performances of this innovative approach, numerous perspectives are possible. First, other evaluation tests must be done to quantify the influence of moving objects on the road. To filter primitives corresponding to these objects, it is proposed to combine our approach with obstacle detection algorithm (ie. from stereovision, laser, or radar processing). Furthermore, to compensate vehicle pitch, a new estimator will be added to apply a real time correction in the projection module. Finally, this approach was originally made to identify the road lanes by using of a multicamera fusion architecture. It is therefore appropriate to continue this multi-cameras study to quantitatively determine the gain provided by the use of other cameras. In addition it will be interesting to study the intrinsic and extrinsic best configuration for this network of embedded optical sensor.

\section{ACKNOWLEDGEMENT}

This work is part of e-future, a 3-year European FP7 research project (project number: 258133) for electric car au- tomation and of CooPerCom, a 3-year international research project (Canada-France). The authors would like to thank the European committee, the National Science and Engineering Research Council (NSERC) of Canada and the National Agency for Research (ANR) of France for supporting these projects.

\section{REFERENCES}

[1] J.-P. Tarel, S.-S. Ieng, and P. Charbonnier, "Using Robust Estimation Algorithms for Tracking Explicit Curves," in ECCV 2002, ser. Lecture Notes in Computer Science, A. Heyden, G. Sparr, M. Nielsen, and P. Johansen, Eds. Springer Berlin / Heidelberg, 2002, vol. 2350, pp. 492-507, 10.1007 3-540-47969-4 33. [Online]. Available: http://dx.doi.org/10.1007/3-540-47969-4 33

[2] R. Sittler, "An optimal data association problem in surveillance theory," IEEE transactions on military electronics, vol. 8, no. 2, pp. 125-139, 1964.

[3] M. Bertozzi and A. Broggi, "Gold: a parallel real-time stereo vision system for generic obstacle and lane detection," IEEE Transactions on Image Processing, vol. 7, no. 1, pp. 62 -81, Jan. 1998.

[4] B. Vanholme, D. Gruyer, S. Glaser, and S. Mammar, "A legal safety concept for highly automated driving on highways," in Intelligent Vehicles Symposium (IV), 2011 IEEE. IEEE, 2011, pp. 563-570.

[5] E. Pollard, D. Gruyer, J. Tarel, S. Ieng, and A. Cord, "Lane marking extraction with combination strategy and comparative evaluation on synthetic and camera images," in Intelligent Transportation Systems (ITSC), 2011 14th International IEEE Conference on. IEEE, 2011, pp. 1741-1746.

[6] D. Gruyer, S. Glaser, and B. Monnier, "Sivic, a virtual platform for adas and padas prototyping, test and evaluation," in FISITA 2010 World Automotive Congress, Budapest, Hungary, 2010.

[7] S. Ieng, J. Vrignon, D. Gruyer, and D. Aubert, "A new multi-lanes detection using multi-camera for robust vehicle location," in Proc. on thee IEEE Intelligent Vehicles Symposium. IEEE, 2005, pp. 700-705.

[8] D. Gruyer, M. Grapinet, and P. De Souza, "Modeling and validation of a new generic virtual optical sensor for adas prototyping," in Intelligent Vehicles Symposium (IV), 2012 IEEE. IEEE, 2012, pp. 969-974.

[9] T. Veit, J. Tarel, P. Nicolle, and P. Charbonnier, "Evaluation of road marking feature extraction," in IEEE 11th International Conference on Intelligent Transportation Systems. IEEE, 2008, pp. 174-181.

[10] E. Pollard, D. Gruyer, J.-P. Tarel, S.-S. Ieng, and A. Cord, "Lane marking extraction with combination strategy and comparative evaluation on synthetic and camera images," in Intelligence Transportation System Conference, 2011.

[11] B. Yu and A. Jain, "Lane boundary detection using a multiresolution hough transform," in Proceedings of the International Conference on Image Processing, vol. 2. IEEE, 1997, pp. 748-751.

[12] S. Ieng, J. Tarel, and P. Charbonnier, "Modeling non-gaussian noise for robust image analysis," in Proceedings of International Conference on Computer Vision Theory and Applications. Citeseer, 2007, pp. 175-182.

[13] B. Flannery, W. Press, S. Teukolsky, and W. Vetterling, "Numerical recipes in c," Press Syndicate of the University of Cambridge, New York, 1992.

[14] Z. Kim, "Robust lane detection and tracking in challenging scenarios," IEEE Transactions on Intelligent Transportation Systems, vol. 9, no. 1, pp. $16-26$, march 2008.

[15] P. Smets, "The combination of evidence in the transferable belief model," IEEE Transactions on Pattern Analysis and Machine Intelligence, vol. 12, no. 5, pp. 447-458, 1990.

[16] D. Gruyer, "Etude du traitement de données imparfaites pour le suivi multi-objets: Application aux situations routières," Ph.D. dissertation, 1999.

[17] C. Royère, D. Gruyer, and V. Cherfaoui, "Data association with believe theory," in International Conference on Information Fusion, vol. 1, 2000, pp. 2-3.

[18] D. Gruyer, C. Royere, and V. Cherfaoui, "Heterogeneous multi-criteria combination with partial or full information," Fusion03 $\{29\}$, p. 1, 2003.

[19] B. Southall and C. Taylor, "Stochastic road shape estimation," in Computer Vision, 2001. ICCV 2001. Proceedings. Eighth IEEE International Conference on, vol. 1, 2001, pp. 205 -212 vol.1. 